\title{
Feature Extraction of Frequency Bands Power Based on Wavelet Packet Decomposition
}

\author{
Enxiang Du, Wei Wang, Chunlin Zhang \\ Department of Arm's Engineering \\ Academy of Armored Force Engineering \\ Beijing china
}

\begin{abstract}
Homing and control signal quality of certain missile is an important information which can reflect system performance and technical conditions of guidance system and it is one of core contents of guidance system detection. The method of feature extraction based on frequency bands power of wavelet packet decomposition is put forward and an illustrative example is proposed in the paper.
\end{abstract}

Keywords-component; control signal; Wavelet Packet Decomposition; feature extraction

\section{DRAWBACK OF Traditional DiagnOSIS METHOD}

Command factor and control signal voltage amplitude are the evaluation index of control signal quality, and command factor and control signal voltage themselves are momentary values which are adopt equalization method. Command factor is just involved with location and polarity of the zero point of the control signal which contains less signal characteristics information and cannot reflect the quality of the whole control signal in the course of Homing and control.

Control signal is pulse width signals, the theory waveform is as Fig. 1 shows. In general cases, control signal cross over the zero point 4 times in a cycle. Traditional method of signal diagnose is to estimate location and polarity of the zero point and amplitude of control signal voltage, which cannot contain all control signal waveform characteristics information. The control signal is not rigorously square signal. The waveform information of rising edge $T_{f}$ and fall edge $T_{c}$, duty ratio, asymmetry of voltage amplitude,etc. are important characteristic information of control signal, which will influence the location of the zero point indirectly. The waveform information of control signals will influence the quality of the control signal.

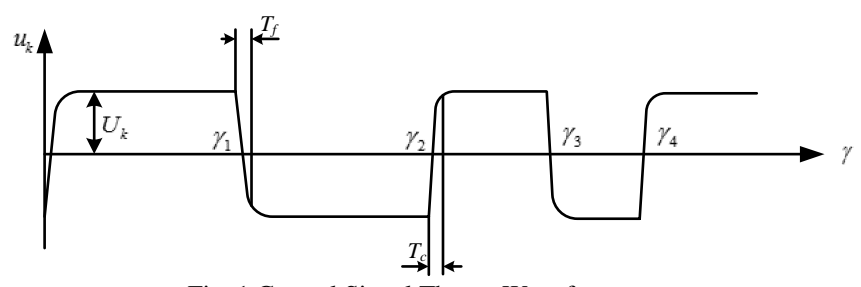

Fig. 1 Control Signal Theory Waveform

\author{
Jingjing Ren \\ LangFang Advanced Technical School \\ Lang Fang He bei, china
}

\section{FEATURE EXTRACTION OF FREQUENCY BANDS POWER}

During to the influence of vehicle vibration environment, electrical impulse and special climatic conditions, the hardware circuit component will age, which cause change of circuit characteristics. With which the waveforms of control signal will also change. For instance: amplitude vibration, change of $\mathrm{T}_{\mathrm{f}}$ and $\mathrm{T}_{\mathrm{c}}$, change of the zero point location,etc. The changes of the waveform information will cause the change of characteristics of some certain frequency, which contains the change of waveform information. Frequency characteristics change is difficult to analyze in timedomain range and Fourier transform and short-time Fourier transform in frequency domain range are two kinds of methods, however, which cannot reach the high resolution requisition of the control signal analysis.

Wavelet packet decomposition is a hot spot of research in signal processing in recent years, which is the linear combination of Wavelet function ${ }^{[1]}$. But it has higher resolution, self-adaptive localization features in time and frequency domain, orthogonality and time-frequency localization property, which is fitter for control signal analysis. As to a certain control signal, it has certain power distribution in the range of specific frequency. When the waveform information is changed by the systematic circuit component aging, the power distribution in the range of specific frequency will change whose essence is the change of enhancement and inhibition effect of every frequency composition of the signal. With the difference transformation rate, this kind of effect is different ${ }^{[2]}$.

Feature extraction of control signal is divided into two courses: Wavelet Packet Frequency Bands Decomposition and Frequency Bands Power Calculation ${ }^{[3]}$.

A. Wavelet Packet Frequency Bands Decomposition

The integral transformation to a certain analog signal:

$$
W_{f}(a, b)=\int_{R} f(t) \psi_{a b}(t) d t
$$

Which is called wavelet transform ${ }^{[4]}$,

$$
\psi_{a b}(t)=|a|^{\frac{1}{2}} \psi(a t-b)
$$


In the formula: $a$ - size parameter

$b$ - translation parameter

$\psi(t)$ is a result of panning and zooming, when a certain condition is satisfied, $\psi(t)$ became allowable wavelet function $^{[5]}$.

In practical application, continuous variable $a$ and $b$ are valued in integer discrete form usually in $\psi_{a b}(t)$, then $\psi_{a b}(t)$ will be showed as formula (3):

$$
\psi_{j, k}^{i}(t)=2^{\frac{j}{2}} \psi\left(2^{j} t-k\right)
$$

In the formula:

$i$-frequency parameter

$j$ - size parameter

$k$-translation parameter.

Corresponding Wavelet Transform become DWT (Discrete Wavelet Transform):

$$
W_{f}(j, k)=\left(f(t), \psi_{j, k}^{i}(t)\right)
$$

DWT is a function of frequency index $j$ and translation index $k$.

Wavelet packet is the linear combination of Wavelet function ${ }^{[6]}$ :

$$
\begin{aligned}
\psi^{2 i}(t) & =\sqrt{2} \sum_{k=-\infty}^{+\infty} h(k) \psi^{i}(2 t-k) \\
\psi^{2 i+1}(t) & =\sqrt{2} \sum_{k=-\infty}^{+\infty} g(k) \psi^{i}(2 t-k)
\end{aligned}
$$

A certain signal $f(t)$ can be decomposed:

$$
\begin{gathered}
f(t)=\sum_{i=0}^{2^{j}-1} f_{j}^{i}(t)=\sum_{i=0}^{2^{j}-1} \sum_{k=-\infty}^{+\infty} c_{j, k}^{i} \psi_{j, k}^{i}(t) \\
c_{j, k}^{i}=\int_{-\infty}^{+\infty} f(t) \psi_{j, k}^{i}(t) d t
\end{gathered}
$$

In the Formula:

$\psi_{j, k}^{i}(t)$-Wavelet packet function, $c_{j, k}^{i}$-Wavelet packet coefficient。

The structure of wavelet packet decomposition is as Fig. 2 shows.

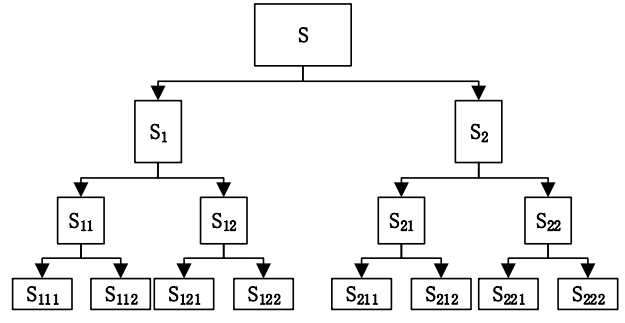

......

Fig. 2 The structure of wavelet packet decomposition

\section{B. Frequency Bands Power Calculation ${ }^{[7]}$}

$f_{j}^{i}(t)$ is the sub-signal of level $j$ and sub-frequency bands $i$ :

$$
E_{j}^{i}=\int_{0}^{T}\left|f_{j}^{i}(t)\right|^{2} d t
$$

In the formula:

$\mathrm{T}$-sampling time

The power of sub-frequency bands:

$$
E_{i}=\sum_{j=1}^{J} E_{j}^{i}
$$

The total power of the signal:

$$
E=\sum_{i=1}^{N} E_{i}
$$

In the formula:

$\mathrm{N}$-number of sub-frequency bands

Frequency bands power distraction vector

$E=\left(E_{1}, E_{2}, \ldots, E_{N}\right)$ can be got by formula $(3-10)$ and ( 3-11), Which is normalized, $E^{\prime}=\left(E_{1} / E\right.$, $\left.\mathrm{E}_{2} / \mathrm{E}, \ldots, \mathrm{E}_{\mathrm{N}} / \mathrm{E}\right)$ can be got.

\section{INSTANCE ANALYSIS}

Control signal voltage $(+220 \mathrm{~V}$ and $-220 \mathrm{~V})$ whose frequency range from $13 \mathrm{~Hz}$ to $15 \mathrm{~Hz}$ can got from Control Box, Launch bracket and Launcher by detecting system. According the sampling laws, sampling frequency is set as $50 \mathrm{~Hz}$. By depressurization, attenuation, isolation, control signal voltage $(+5 \mathrm{~V}$ and $-5 \mathrm{~V})$. By A/D transform, control instruction can be got ${ }^{[8]}$.

By investigation from the ordnance factory, 137 groups control signal voltage can be got. Among them, 53 groups are fault signal data after measuring. Any one of fault signal data and non-fault signal data are used to emulation by MATLAB. The above-mentioned two groups of control signal data waveform are as Fig. 3 and 4 shows. 


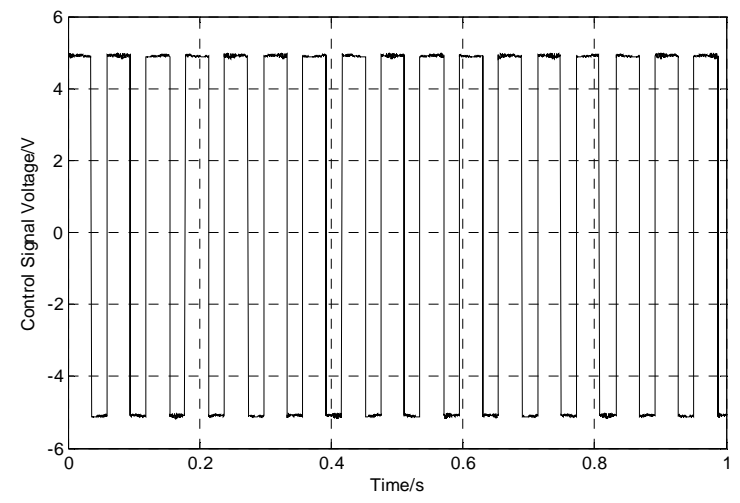

Fig. 3 Waveform of fault signal

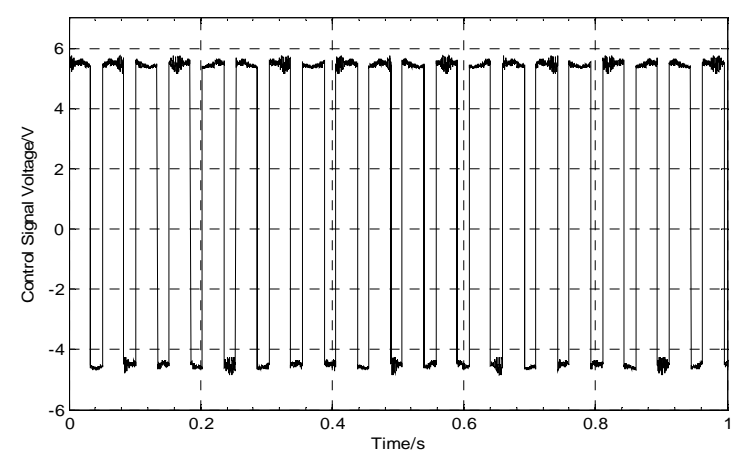

Fig. 4 Waveform of non-fault signal

Calling wpdec and wprcoef function in MATLAB (wavelet packet decomposition and reconstruction function $)^{[9]}$, above-mentioned two groups of control signal can be conduct on 3 layers frequency bands decomposition and divided into 8 frequency bands which is about $3.125 \mathrm{~Hz}$ of bandwidth within the range of $25 \mathrm{~Hz}$. 8-dimensional frequency bands power distraction vector (dimension is $1 * 10^{5}$ ) are as Table I . shows.

TABLE I. TABLE TyPe Styles

\begin{tabular}{|c|c|c|}
\hline $\begin{array}{c}\text { Frequency Bands } \\
\text { Power }\end{array}$ & Non-fault Signal Data & Fault Signal Data \\
\hline $\boldsymbol{E}_{1}$ & 0.1463 & 0.4252 \\
\hline $\boldsymbol{E}_{2}$ & 0.5968 & 0.3123 \\
\hline $\boldsymbol{E}_{3}$ & 7.2914 & 7.0572 \\
\hline $\boldsymbol{E}_{4}$ & 1.3806 & 2.2503 \\
\hline $\boldsymbol{E}_{5}$ & 0.0478 & 0.0528 \\
\hline $\boldsymbol{E}_{6}$ & 0.0642 & 0.0641 \\
\hline $\boldsymbol{E}_{7}$ & 0.1588 & 0.1731 \\
\hline $\boldsymbol{E}_{8}$ & 0.0979 & 0.1410 \\
\hline
\end{tabular}

Corresponding power histograms are as Fig. 5 and 6 show. As to a certain control signal, which has certain power distribution in the range of specific frequency. When the waveform information is changed by the systematic circuit component aging, the power distribution in the range of specific frequency will change.

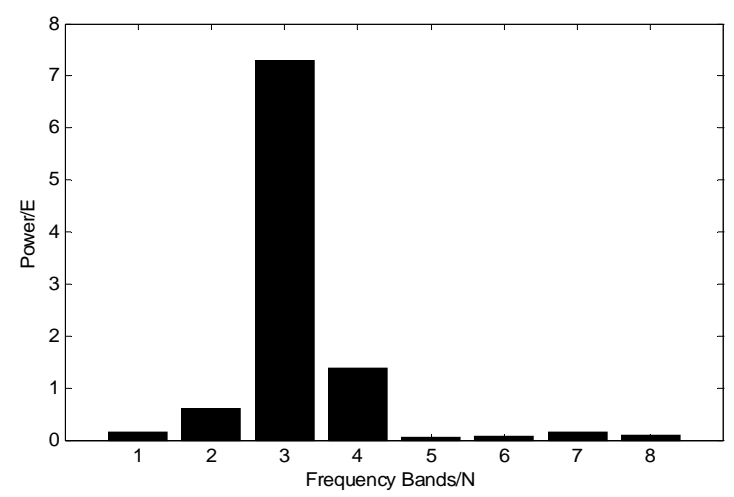

Fig. 5 Frequency bands power distraction of non-fault signal

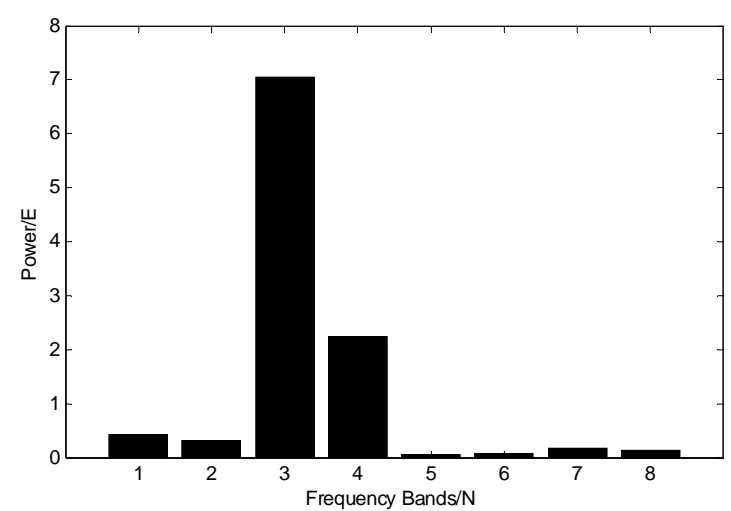

Fig. 6 Frequency bands power distraction of fault signal

\section{CONCLUSION}

From frequency bands power distraction histograms, the main power of control signal is concentrated on the 3rd and the 4th frequency bands, that is to say, the main frequency range of $13 \mathrm{~Hz}$ to $15 \mathrm{~Hz}$ of control signal. Frequency main bands power of fault signal is weakened and shifts to other frequency bands. According to the basic prerequisite of the conservation of power in the transform, power only be shifted in every frequency band and will not disappear. The essence of the frequency bands power transfer reflects the course of the waveform information of control signal, which caused by the systematic circuit component aging. So the method of feature extraction of frequency bands power based on wavelet packet decomposition is applicable and reasonable.

\section{REFERENCES}

[1] Daubechies I. Orthonormal bases of compactly supported wavelets[J]. Comm, Pure Appl. Math, 1988:11-41.

[2] Daubechies I. Ten Lectures on Wavelets[C] CBMS-NSF Regional Conf: Series in Appl. Math. Vol. 61. Philadelphia: Society for Industrial and Applied Mathematics, 1992.

[3] Fu Xiao, Chen liuwei, Sun Yanjun. Threshold de-noising algorithm on [J]. computer programming technology based on wavelet analysis and maintenance, 2012, (4). 
[4] He Wei, Wang Yi, Li Huaxing. Adaptive redundant second generation wavelet in signal to noise in the application of [J], the mining machinery, 2012,40 (2).

[5] Joseph O, Raghuveer M R. Algorithms for wavelets to match a specified signal[J]. IEEE Transaction on Signal Processing,2000, 48(12): 3 395-406.

[6] S. Mallatetal.Characteristics of signals from multiscale edges.IEEE Trans.on PAMI,1977;7.
[7] Jie Liang \& Thomas W. Parks. A Translation-invariant Wavelet Representation Algorithm with Application.IEEE Trans on SP,1996;2.

[8] FU MING. The application of decreasing noise by wavelet transform

[9] Dorigo M,Maniezzo V,Colomi A. Ant System:Optimization by a Colony of Cooperation Agents[J].IEEE Transactions on Systems,Man,and Cybemetics,1996,26(1):29-4 